\title{
From Small to Big: Smartwatch Use in Mitigating COVID-19 - Understanding User Experience from Social Media Content Analysis
}

\author{
Jianwei Lai \\ Illinois State University \\ jlai12@ilstu.edu
}

\author{
Lina Zhou \\ The University of North \\ Carolina at Charlotte \\ lzhou8@uncc.edu
}

\author{
Kanlun Wang \\ The University of North \\ Carolina at Charlotte \\ kwang17@uncc.edu
}

\author{
Dongsong Zhang \\ The University of North \\ Carolina at Charlotte \\ dzhang15@,uncc.edu
}

\begin{abstract}
Smartwatches offer both functions and convenience that can have great potentials for technological interventions. Despite widespread discussion of technological interventions for COVID-19, smartwatch use has received little attention in the literature. This research aims to fill the literature gap by providing a broad understanding of smartwatch use for COVID-19 mitigation. We investigate smartwatch use through content analysis of the data collected from two social media platforms. The method allows us to draw on user experience beyond technological features and functions. In addition to functions, we also identified the concerns of using smartwatches for mitigating COVID-19. Furthermore, we uncovered both similarities and differences between the different social media platforms in terms of functions and concerns of smartwatch use. Our findings have implications for various stakeholders of the smartwatch technology and for mitigating the impact of the pandemic.
\end{abstract}

\section{Introduction}

Technologies are playing an increasingly important role in combating COVID-19. Smartwatches, as a type of wearable device, are convenient for daily use. They allow users to keep track of their overall health and physical activities, check the weather and time, listen to music, and receive emails and text messages, etc. According to a report by the Pew Research Center, about 21 percent of Americans regularly use a smartwatch or fitness tracker [1]. Gartner Inc. predicted that the global spending on wearable devices would total $\$ 81.5$ billion in 2021, driven by the rise of remote work and interest in health monitoring [2]. By enabling continuous health monitoring, wearable devices have been used to detect the onset of infectious diseases, such as respiratory viral infection [3]. Thus, smartwatches have great potentials for mitigating COVID-19.

Despite widespread exploitation of technological interventions for COVID-19, smartwatches have received much less attention in the literature as a potential technology for fighting the pandemic. Early detection of COVID-19 is not only important for patients to get early treatment, but also helpful for mitigating the spread of the virus [4]. Quer et al. [4] collected smartwatch and activity tracker data from more than 30,000 participants and found that a model considering both pandemic symptoms and smartwatch sensor data performed better in detecting COVID-19 cases than a model without sensor data. Mishra et al. [5] analyzed retrospective smartwatch data and found that $63 \%$ of COVID-19 cases could have been detected based on users' extremely elevated resting heart rate even before symptoms appear. Smartwatch data can help detect COVID-19 infection when patients are presymptomatic [6], which may provide a convenient way of detecting COVID-19 cases early. Those studies used smartwatch data for the purpose of early detection. However, they did not consider other functions of smartwatches in combating the pandemic. Apart from the benefits, smartwatch use may also raise concerns, which have not been examined in the literature. There is another stream of studies on the role of mobile apps in COVID-19 intervention. Nevertheless, most of them (e.g., [7-11]) have focused on the technical features and functions of mobile apps, and those apps are not specifically limited to smartwatches.

This study aims to fill the above-mentioned literature gaps by providing a broad understanding of smartwatch use for the COVID-19 mitigation. Specifically, the study is focused on three research questions: What are the functions of smartwatches in mitigating COVID-19? Do users have concerns about using smartwatches in coping with the pandemic? If so, what are those concerns? Does the social media platform have an effect on the functions, concerns, and topics of smartwatch use in mitigating COVID-19? We answer the questions by analyzing the content of data collected from two social media platforms.

This research makes the following main contributions. First, to the best of our knowledge, our 
study is the first that systematically investigates the use of smartwatches for mitigating the impact of COVID19. Our proposed schema for analyzing smartwatch use in social media data, which covers the aspects of both functions and user concerns, can provide guide for future research and development efforts. Second, using the publicly available user-generated data lends ecological validity to our findings. The new functions and concerns of smartwatch use for COVID-19 mitigation identified in this study may be generalized to other types of technological interventions. Third, this is the first study that reveals the similarities and differences between two popular social media platforms in the smartwatch use for COVID-19 mitigation.

\section{Related work}

Based on an extensive literature review, we organize the related work from general to specific topics.

\subsection{Non-medical interventions for COVID-19}

Due to the high contagion of COVID-19, it is crucial to deploy various intervention solutions to mitigate its transmission and ultimately save lives. The Department of Homeland Security Science and Technology Directorate in the United States has developed a non-medical intervention framework with support from other federal agencies to help individuals, households, and organizations to protect themselves, their families, and people in their communities and institutions [12]. The framework [12] identifies four types of intervention, including 1) materiel, 2) social and behavioral, 3) strategy, policy, and program, and 4) economic and resource. Materiel intervention is referred to as providing necessary equipment, supplies, physical systems, and structural modifications, such as Personal Protective Equipment and hand sanitizers. Social and behavioral intervention refers to action-based changes to mitigate the spread of COVID-19, such as social distancing and hand washing. Strategy, policy, and program intervention focuses on providing guidance to direct or inform actions to mitigate the spread of COVID-19 by governing organizations. Economic and resource interventions are intended to mitigate health, safety, and financial risks and consequences of a pandemic and promote a faster recovery (e.g., personal subsidies, unemployment benefits).

\subsection{Functions of technological intervention}

Technology cuts across multiple types of nonmedical interventions from the above framework. For instance, technologies can be used to support individuals' behavioral change, enforce a government's public health policies and guidelines, and enable touchless system interactions. In terms of functions, technologies have been used to support testing [13], surveillance and detection (e.g., thermal vision camera [14], tracing (e.g., geolocation tools [15] and tracing app [16]), and prediction of COVID-19 [17]. In addition, technology has also been used to support people with special accessibility needs [18] or underlying health conditions [19], health service delivery [20], and training community practitioners [21].

New technologies have been developed or proposed and existing technologies have found their novel applications in combating COVID-19. Some of these technologies include drones and robots, blockchain, geolocation tools, mobile apps, wearable sensors and/or IoT, Bluetooth, 3D printing, artificial intelligence, 5G network, telehealth, etc. For instance, drones and robots have been used to monitor people's compliance with the lockdown policy to contain the disease [22]. However, the understanding of the impact of technological interventions on health and non-health outcomes during the pandemic is very limited partly because of delayed implementation [22]. Technological intervention for COVID-19 is also facing adoption barriers such as the invasion of privacy [23].

\subsection{COVID-19 related mobile apps}

Smartwatches are often used when paired with smartphones, which is reflected by the data collection methods used in some previous studies (e.g., [4, 24, 25]). Additionally, mobile apps are a very active area of development for combating COVID-19.

Mobile apps have been examined from different dimensions, such as data collection technology (e.g., Bluetooth, GPS, QR codes), mobile platform (e.g., iOS, Android), data organization (e.g., centralized vs. decentralized), mandatory vs. voluntary, data entry (automated or manual), and functionality (e.g., tracing contacts, symptom monitoring, online consultation with health authorities) [7, 26, 27]. Some of the studies directly reviewed COVID-19 mobile apps available in Google Play and the Apple App Store [7-10]. Other studies (e.g., [27]) reviewed mobile apps discussed in related research articles. However, these studies mainly focused on the technical features and functions of COVID-19 apps, which may not reflect user experience, and more importantly, the context of those studies are not smartwatches.

Weiß et al. [28] collected information of contact tracing apps in different countries by drawing from academic publications, public government data, grey literature, and media. They overlooked user experience 
toward the specific type of mobile apps. Kaspar et al. [29] used a survey to investigate participants' motivations to use a contact tracing app and a data donation app. Another study also used surveys [30] to investigate the factors that affect the adoption of a COVID-19 tracing app. Elkhodr et al. [31] analyzed users' reviews of contact tracing apps from app stores. The focus of the study was the underlying technology and data collection and destruction. However, those studies did not consider other functions of mobile apps and user concerns about the technology.

\subsection{Smartwatch use for combating COVID-19}

A few studies focused on providing non-medical intervention of COVID-19 with smartwatches. For example, Sudharsan et al. [32] continuously monitored human arm and hand movements using a wearable device to warn device users before their hands touched their faces. In addition, iWash [33], a smartwatch handwash system, was developed to remind users of washing their hands when they come back home. Studies have collected smartwatch data over time to detect/predict COVID-19 cases. For example, Quer et al. [4] and Mishra et al. [5] found that smartwatch sensor data can help with detecting COVID-19 positive cases for both symptomatic and pre-symptomatic patients.

Several studies looked into providing smartwatchbased telemedicine during the pandemic. Strik et al. [34] investigated using Apple Watch ECG data to provide remote monitoring for QT prolongation of COVID-19 patients. Chinitz et al. [35] presented a case of using an Apple Watch to monitor a COVID-19 outpatient for arrhythmias and QT prolongation when she was isolated at home. Smartwatches were also used to facilitate telemedicine for severe aortic stenosis patients after undergoing a transcatheter aortic valve replacement procedure [36]. Another group of related studies used smartwatches as a lens to examine the impact of the pandemic on people's well-being or physical activities. For instance, Niela-Vilén [37] used longitudinal smartwatch data to investigate the daily well-being patterns of pregnant women before and during the pandemic, and they found variability in heart rate, elevated stress levels, and change in sleep. Ueafuea et al. [38] discussed the impact of COVID-19 on people's mental health and the potential of providing psychological support with mobile and wearable devices. These studies provide evidence for the various functions of smartwatches in mitigating the effect of the pandemic. However, individual studies were application-specific and users were passive providers of raw sensor data.

This study aims to address the limitations of the reviewed studies by investigating the smartwatch use for COVID-19 mitigation in a natural setting. We analyzed the content of the collected social media data to gain broad and deep insights into the related functions and user concerns. Moreover, we compared the analysis results between two social media platforms to understand the possible platform effect. Furthermore, we analyzed the topics or themes of the social media content to better understand related smartwatch issues.

\section{Methods}

We answer our research questions by collecting and analyzing social media data from two platforms.

\subsection{Data collection}

We selected Reddit and Twitter as the social media platforms for data collection for two main reasons. First, they represent different types of platforms. Twitter is a microblogging website, whereas Reddit is a social bookmarking community. Reddit allows users to discuss topics in which they are interested anonymously, which makes communication more flexible and unconstrained [39]. These different characteristics of platforms allowed us to understand their possible impacts on users' smartwatch use in response to COVID-19. Second, both are popular platforms for online communities. For instance, Twitter and Reddit have more than 300 and 430 million active users on a monthly basis, respectively [40, 41].

Following recent COVID-19 studies [42-44], we first used "COVID", "COVID-19", "Coronavirus", "SARS-COV-2", "Disease", "pandemic" and their different variations and combinations of those keywords to collect relevant posts from Reddit on a daily basis, and we then applied the snowballing approach to scrape all corresponding comments/replies to the original posts. Next, we performed data filtering using smartwatch-related key terms such as "smartwatch", "smart watch", "sw", and associated model names (e.g., wear os, pebble, Fitbit). Finally, we removed redundant content presented as subsets or replicates of previous posts across different post days. The collection of tweets

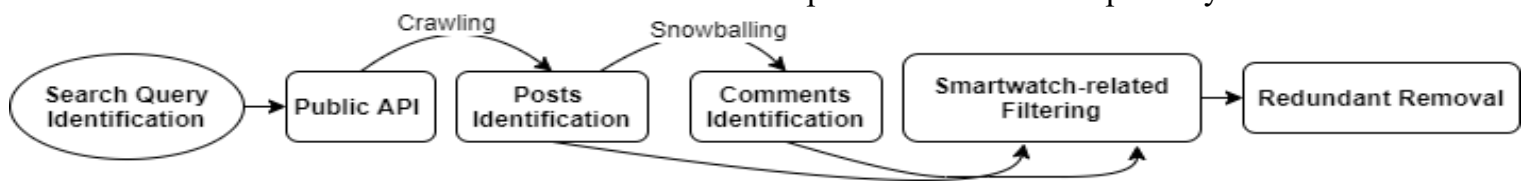

Figure 1. The Procedure for Data Collection and Preprocessing 
followed a similar process. The original tweets were collected through Twitter API using the same set of key terms as we used for the Reddit data collection. We removed all the retweets and only kept the original tweets in the data collection. To comply with the privacy policy of the platforms, we only collected publicly available information. The data collection process is described in Figure 1.

\subsection{Content analysis}

Content analysis allows us to make valid inferences from data in a specific context [45]. It is a powerful and versatile tool that can be used to analyze textual, visual, and audio data [46]. To perform content analysis, we need to select data samples and prepare a coding schema. We performed random sampling to select posts from the collected social media data (see Section 3.1). The content analysis went through three stages: coding schema creation, schema refinement, and data analysis and validation.

The primary goal of the first stage was to prepare the post annotation schema. During this stage, we randomly selected 2,000 tweets from the Twitter dataset and applied open coding to induct themes from the tweets. Open coding is the first-level coding, which is used by researchers to identify concepts and themes for categorization [47]. Two coders who had been actively involved in mobile computing research for over a decade went through the entire sample sets independently. It is worth noting that many of the tweets were not relevant to COVID-19, such as ads for mobile apps, news sharing, and tweets only containing relevant terms in their hashtags. Those posts were excluded from the analysis. Based on the two sets of coding results, the two coders met and discussed the labels, which led to the creation of a coding schema.

We validated the coding schema in the second stage. During this stage, we randomly sampled 50 posts from the Reddit dataset. The same two coders and the third coder analyzed those posts independently using the developed coding schema while allowing for creating new labels. We consolidated the coding results of three coders, and then the coders met to resolve any coding differences through discussion. This led to a refinement of the previous coding schema, including merging or reclassifying existing items and creating new items.

In the last stage, we randomly selected another 275 Reddit posts and 250 tweets from the dataset used in the first stage of content analysis. We assigned each post to two coders who analyzed them independently. Some of the posts were still found to be noisy and were thus removed. The final dataset consisted of 225 Reddit posts and 210 tweets. We then followed the same consolidation and discussion steps as we did in the second stage. Further, we validated the coding results through inter-rater reliability analysis, which quantifies the degree of agreement between multiple coders who conduct independent observational ratings on qualitative or quantitative data [48]. The inter-rater reliability results are presented in Table 1.

\subsection{Topic modeling}

Latent Dirichlet Allocation (LDA) [49] has been widely applied to modeling topics, text mining, social media analysis, and information retrieval. It has been proven to be one of the most reliable and valid methods for discovering unsupervised themes in communication research [50]. We deployed the LDA method with the following four steps: 1) pre-processing of the social media text; 2) selection of model parameters, including the number of topics to be generated; 3) evaluation of the model's reliability; and 4) interpretation of the topics generated. Given that the LDA method relies on the bagof-word assumption, the word order and phrases are also critical for capturing the meaning of textual content in many text mining tasks [51]. Hence, we implemented a probabilistic model using n-grams (bi-grams and trigrams) to extract and retrieve meaningful topics.

\section{Results}

A list of functions and concerns of using smartwatches for coping with COVID-19 and their frequency counts are reported in Table 1. The table also shows the percentage of agreement for each of the functions and concerns from the content analysis results.

Table 1: Frequency Count and Inter-rater Agreement of Functions and Concerns

\begin{tabular}{|c|c|c|c|c|c|}
\hline & \multirow[b]{2}{*}{ Category } & \multicolumn{2}{|c|}{ Reddit } & \multicolumn{2}{|c|}{ Twitter } \\
\hline & & $\begin{array}{l}\text { Frequency } \\
\text { count }\end{array}$ & $\begin{array}{l}\text { Agreement } \\
\text { percent }\end{array}$ & $\begin{array}{l}\text { Frequency } \\
\text { count }\end{array}$ & $\begin{array}{l}\text { Agreement } \\
\text { percent }\end{array}$ \\
\hline \multirow{7}{*}{ Function } & tracking & 71 & $86 \%$ & 88 & $80.95 \%$ \\
\hline & $\begin{array}{l}\text { detection \& } \\
\text { prediction } \\
\end{array}$ & 32 & $94.55 \%$ & 81 & $80.00 \%$ \\
\hline & $\begin{array}{l}\text { preventative } \\
\text { intervention }\end{array}$ & 16 & $94.73 \%$ & 18 & $94.29 \%$ \\
\hline & \begin{tabular}{|l|} 
monitoring/ \\
rehabilitation
\end{tabular} & 9 & $94.73 \%$ & 6 & $91.43 \%$ \\
\hline & $\begin{array}{l}\text { research } \\
\text { participation }\end{array}$ & 4 & $98.48 \%$ & 11 & $96.67 \%$ \\
\hline & reporting & 1 & $98.91 \%$ & 4 & $97.14 \%$ \\
\hline & telehealth & 0 & $100.00 \%$ & 8 & $94.29 \%$ \\
\hline \multirow{4}{*}{ Concern } & privacy & 33 & $97.82 \%$ & 2 & $36.19 \%$ \\
\hline & \begin{tabular}{|l} 
false \\
positives
\end{tabular} & 19 & $93.52 \%$ & 3 & $81.43 \%$ \\
\hline & $\begin{array}{l}\text { information } \\
\text { overload }\end{array}$ & 2 & $100 \%$ & 0 & $94.29 \%$ \\
\hline & usability & 1 & $100 \%$ & 9 & $100 \%$ \\
\hline
\end{tabular}




\subsection{Functions}

4.1.1. Tracking. It is shown from Table 1 that the most common function of smartwatches during the pandemic is tracking physical activities, such as general workouts, walking, and sleeping activities. The following posts are examples of this function:

"@fitbit shows one adverse \#covid19 effect: Number of \#step decreased over the world. Physical distancing is not a reason to stop to do [doing] \#exercise / \#move. Don't forget physical \#inactivity is the 4th cause[s] of mortality (@WHO)."

"Interesting mobility reduction figures per country $v$ [the] same date in 2019. Based on real GPS data from Fitbit activity tracker (now a Google property)."

4.1.2. Detection/prediction. The second most common general function is detection/prediction. The detection/prediction function focuses on the use of biological data to detect COVID-19 contracts or predict potential cases and tracking contracts. There has been an increase in the novel applications of wearable technology like smartwatches for the detection and prevention of COVID-19 during the pandemic, such as predicting potential cases and early warning of related symptoms. Some representative posts collected are shown below:

"The app gathers vital signs from volunteers wearing smartwatches or fitness trackers - including pulse, temperature [,] and sleep - to analyse whether they are symptomatic of the flu-like illness."

"Fitbit aims to develop an algorithm that can highlight potential cases before symptoms start."

We further identify two main sub-functions of detection/prevention: monitoring signs/biological signals and tracing contacts. The following are representative samples for signs/biological signals and tracing contacts.

- Signs/biological signals

"A cool alternative I heard about that occurs before fevers is that people often have heart rate increase before [a] fever develops so tracking heart rate with f[F]itbit might help catch more presymptomatic[s] [cases]."

- Tracing contacts

"If you're wearing it, you walk straight into shopping malls, public transport [,] etc. If you don't have one, you scan Safe Entry or show TraceTogether on your phone. Or in the strictest implementation, you have to have it on in public, just like a mask."

"We can use digital markers to identify a cluster where there is a potential \#COVID19 emerging outbreak. This could be done via active means of body temp or passive thru smartwatches. Over 100 million
Americans have a smartwatch or fitness band that captures resting heart rate $5 / x$."

4.1.3. Preventative intervention. The application of smartwatches for the preventative intervention of COVID-19 is also commonly mentioned in social media posts. CDC and WHO have recommended many nonmedical interventions over time, such as washing hands. The preventative intervention was mainly about preventing transmission through direct contact and promoting social distance. We further identify two subcategories for the function of preventative interventions, including (direct) transmission prevention and social distancing. We provide representative examples for each of the subcategories below:

- (Direct) transmission prevention

"Another interesting feature is that the Apple Watch will remind you to wash your hands when you've returned."

“...technology to help fight corona[-]virus. Just an idea, but maybe we can program our smartwatches and fitness bracelets to vibrate and warn us every time we are going to reach our face with a hand."

- Social distancing

"I created an app called Keep Space which uses Bluetooth to detect if someone is nearby, also works for kittens with a f[F]itbit."

4.1.4. Monitoring/rehabilitation. Another interesting and novel application of smartwatches is monitoring related symptoms and facilitating rehab after contracting COVID-19. Here are some examples.

"My heart rate spikes with palpitations. I'm noticing bouts of increased body temperature, increased symptoms, and increased heart rate simultaneously. I don't know what to make of it yet, but being in data analytics, it's intriguing. I believe we're dealing with a very "sneaky" virus that is morphing and hiding to do the most damage. My suggestion is to keep your Fitbit or smartwatch on you tracking [to track] these symptoms. Take your temp frequently and keep a journal."

"Being on the edge of death felt pretty real to me ;) My Rehab has actually been going really well. I'm about to hit 30days of closing all my Apple Activity rings (every single day). My Apple Watch told me I walked 46.7 miles in the past 7 days..."

4.1.5. Research participation. The following sample posts are related to research participation.

"Fitbit gives users the option to support Covid-19 research."

"If you have a f[F]itbit, you can opt into the COVID[-]19 data study within the phone app." 
4.1.6. Reporting. Reporting COVID-19 cases and symptoms via smartwatches is evidenced by the following post:

"The Robert-Koch-Institut (\#RKI) has published a free app for smartwatches and fitness wristbands, which should bring information about the spread of the \#Coronavirus in \#Germany. With the help of the app[,] it is possible to see if there are any signs of infection."

"Google using \#coronavirus outbreak to deploy [ ] deep [ ] state surveillance app called "Corona Waze" and using "social citizen reporting" like China with automatic alerting of agencies using FitBit Biometrics[. ]Using nationwide cameras to identify offenders."

4.1.7. Telehealth. Telehealth has become particularly attractive during the pandemic. This type of posts is not common but quite distinctive. Here are the sample posts:

"\#Wearables Giant Fitbit rolled out new features where its App Users will now be able to connect with a doctor virtually through a membership plan, access information on exercising indoors apart from [the] important information on \#COVID19 from WHO."

"Excited to partner with the amazing@fitbit team to provide millions of users virtual primary care to make it seamless to get access to the best physicians in the country - now more important than ever!"

\subsection{Concerns}

While smartwatches play various roles in combating COVID-19, social media users also expressed various concerns about using them. We grouped the concerns into four main categories: privacy, false positives, information overload, and usability concerns. Some sample posts of each category are shown below:

- Privacy

"When Google bought Fitbit, people were concerned with mass tracking. Is that happening so far?"

“... I figure $\mathrm{O}_{2}\left[\mathrm{O}_{2}\right]$ sat shouldnt[shouldn't] be a privacy issue like heart rate might be."

\section{- False positives}

"You'd get too many false positives or things you can't diagnose well."

\section{- Information overload}

"It might need to hide some details so clinics don't get flooded with hypochondriacs, but it could be a net benefit to public health if handled well."

- Usability

"Apple Watch can remind you to wash your hands when you get home, but I don't see where to enable/disable this and can't get it to work."
As expected, privacy is one of the major concerns with using smartwatches. Like other wearable devices, smartwatches can collect a variety of data about a user's activities, self-entered data, and their interactions with smartwatches. Current privacy policies for many fitness tracking apps allow users' data to be shared with others [52]. Concerns have been expressed about who can and should have access to this data.

As shown in Table 1, users of both Twitter and Reddit showed similar interests in the functions of smartwatches. They all identified "tracking" as the main function, followed by "detection \& prediction" and "preventative intervention". In addition, "privacy" was the top concern of users on Reddit, while Twitter users expressed most concerns on the usability of smartwatches. "False positives" was the second most concern of users on both platforms. In addition, Twitter users seem more interested in the "detection \& prediction" function, given that a much greater number of tweets were commented on that function.

\subsection{Content analysis}

We extracted bigrams and trigrams from the social media content in our data collection. The top- 5 bigrams and trigrams are listed in Table 2. A comparison in terms of bigrams and trigrams between the two social media platforms reveals both similarities and differences. We further reported the topic modeling results and topic labels in Table 3.

Table 2. Top-5 Bigrams and Trigrams

\begin{tabular}{|c|c|c|}
\hline & Reddit & Twitter \\
\hline \multirow{5}{*}{ Bigrams } & sensor, data & fitness, trackers \\
\hline & self-reported, symptoms & help, detect \\
\hline & helmet, gloves & wearables, like \\
\hline & identify, cases & heart, rate \\
\hline & activity, tracker & launches, app \\
\hline \multirow{5}{*}{ Trigrams } & activity, tracker, data & $\begin{array}{l}\text { fitness, trackers, } \\
\text { help }\end{array}$ \\
\hline & \begin{tabular}{|l|} 
self-reported, \\
symptoms, diagnostic
\end{tabular} & trackers, help, detect \\
\hline & symptom, sensor, data & $\begin{array}{l}\text { app, monitor, } \\
\text { coronavirus }\end{array}$ \\
\hline & $\begin{array}{l}\text { develop, wearable, } \\
\text { presymptom }\end{array}$ & $\begin{array}{l}\text { help, detect, } \\
\text { coronavirus }\end{array}$ \\
\hline & $\begin{array}{l}\text { wearable, presymptom, } \\
\text { detectors }\end{array}$ & $\begin{array}{l}\text { launches, app, } \\
\text { monitor }\end{array}$ \\
\hline
\end{tabular}

Based on our observations of the generated topic, we identify four major topics:

$\mathrm{T}_{1}$. the use of smartwatches to keep track of daily activities (e.g., at-home workout, walking steps),

$\mathrm{T}_{2}$. the implementation of sensor data or vital signs (e.g., heart rate) to detect the presymptomatic cases of COVID-19, 
Table 3. Top-5 Topics and Their Words

\begin{tabular}{|l|l|l|}
\hline \multirow{5}{*}{ Reddit } & Words & Topics \\
\cline { 2 - 3 } & Apple Fitbit like new use pulse used phone ox good & $\mathrm{T}_{2}$ \\
\cline { 2 - 3 } & like Fitbit symptoms heart rate people days day percent work & $\mathrm{T}_{2}$ \\
\cline { 2 - 3 } & people apple bought bank media app study phone google years & $\mathrm{T}_{2}$ \\
\cline { 2 - 4 } Twitter & $\mathrm{T}_{3}$ & $\mathrm{~T}_{4}$ \\
\cline { 2 - 4 } & Fitbit coronavirus app data physical health wearables impact research users & $\mathrm{T}_{1}$ \\
\cline { 2 - 3 } & \begin{tabular}{l} 
Stay[-]home social[-]distancing check creative distancing woman 2020 social awesome meters \\
\cline { 2 - 3 }
\end{tabular} & $\mathrm{T}_{1}$ \\
\cline { 2 - 3 } & coronavirus Fitbit app apple germany smartwatches using help day amp & $\mathrm{T}_{3}$ \\
\cline { 2 - 3 } & $\begin{array}{l}\text { Fitbit apple like coronavirus trackers detect fitness wearables help heart } \\
\text { m[-]health babe }\end{array}$ & $\mathrm{T}_{1}$ \\
\cline { 2 - 3 }
\end{tabular}

$\mathrm{T}_{3}$. launching COVID-19 related apps (e.g., tracetogether) for patient monitoring and rehabilitation,

T4. smartwatch-related research on COVID-19 (e.g., announcing a research study and recruiting participants for an experiment).

Given that a wide range of personal sensitive data are stored on smartwatches, it is not surprising that smartwatch users raised privacy concerns with regard to the process of data storage, data analysis, and reporting. However, the instances of false positives, information overload, and usability seem to be intangible given the limited occurrence of those topics.

According to the results, it seems that Twitter users prioritized the use of smartwatches as a tracker, especially tracking their biological signals (e.g., heart rate) during fitness activities. Then, detecting or monitoring the disease by launching apps becomes the secondary request. Despite that a considerable number of users from Reddit have a primary focus on detecting disease-related symptoms by leveraging sensor data, launching pandemic related apps and leveraging research resources could become a supplementary function to support the mitigation of the pandemic.

\section{Discussion}

Through content analysis, we investigated both functions and concerns about the smartwatch use during the pandemic. Specifically, we identified five main smartwatch functions related to the pandemic, including tracking physical activities, preventative intervention, detection and prediction, monitoring for rehabilitation, reporting, and research participation. For some of the main functions, we further classified them into two or more types of specific sub-functions. In addition, we identified four main concerns, including privacy, false positives, usability, and information overload.

Through social media analytics, we identified numerous relevant topics by implementing bigrams, trigrams, and LDA topic modeling techniques. Among them, the dominant topics are about functionalities of smartwatches for health and wellness during the pandemic. It is worth noting that users of smartwatches have actively engaged in interacting with smartwatches for a variety of purposes. In particular, using a smartwatch to estimate the amount of at-home exercise provides a great alternative for those who normally recreate outdoors. Smartwatches offer an efficient and effective way of using vital signs for not only detecting presymptomatic cases but also being suitable for monitoring, rehabilitation, and self-reporting. It is of critical benefit that smartwatches users can receive realtime informative prompts in this difficult time. Moreover, it is common that smartwatch users concern with data security and privacy. However, the disclosures are relatively intangible. Despite that the major themes generated from the two platforms indicate that the two platforms are preferential with the tracking functionality of smartwatches, the path for detecting the symptoms of COVID-19 varies from launching apps to leveraging sensor data. 
This research makes several main contributions. First, mobile apps can run on smartphones and tablets as well as smartwatches. It remains unclear how smartwatches are being used during the pandemic. This study investigated the functions and concerns of smartwatch use during the pandemic for the first time. Second, unlike previous studies with mobile apps that collected data from descriptions of mobile apps, this research adopted a data-driven approach by performing content analysis of social media data. The latter approach is expected to reflect the actual use of smartwatches better. Third, we collected data from two different social media platforms, which is the first study allowing us to gain additional insights into the smartwatch use for COVID-19 from a platform perspective. Fourth, we uncovered new functions of smartwatch use such as monitoring (for rehabilitation), detection and prediction, and preventative intervention. Moreover, we identified new functions such as direct transmission prevention as part of the preventative intervention function. Last but not least, we not only examined concerns about using smartwatches for combating COVID-19, but more importantly, revealed several concerns besides privacy that have never or rarely been identified in the literature, including false positives, information overload, and usability issues.

The implications of our study can be summarized from several different perspectives. From a smartwatch manufacturer's perspective, our study provides a way for developers to determine what functions of a smartwatch are likely to be used and how to leverage such information to design better smartwatches apps and identify how different types of functions affect the smartwatch use during the pandemic. In addition, our method has the potential to provide smartwatch manufacturers with empirical evidence for improving smartwatch and mobile app design. From a smartwatch user's perspective, our results enable users to not only find a set of functions or specific functions that are available in smartwatches to meet users' needs for combating COVID-19 but also alert users about potential concerns that may arise from adopting those smartwatch functions. From a social media platform's perspective, our proposed categorization schema for smartwatch functions and concerns pertaining to COVID-19 can provide social media platforms with guidance on how to better organize and index usergenerated content to improve the relevance and value of the platforms to the pandemic. From a healthcare service provider's perspective, our findings can provide insights on COVID-19 mitigation, for example, enabling early detection of infection with evaluated resting heart rates and providing timely intervention.

Our research has limitations that could serve as areas for future research. First, smartphones are often used in combination with smartwatches. It would be interesting to repeat the same study with smartphones and compare whether and how the functions of smartwatches and smartphones may complement each other. Second, because our sample size is relatively small, some functions of smartwatches have low occurrence frequencies. Increasing the sample size to reach saturation may identify additional functions or concerns. Third, we assume that the proposed classification schema is generalizable across different social media platforms, which need to be validated in a future study. Fourth, social media users can have diverse backgrounds and expertise, which offer different perspectives regarding using smartwatches for COVID19 mitigation. We used the public information from the two social media platforms in this study, which did not provide detailed information on the data sources. Future research may look into social media sources to identify and compare the perspectives from different stakeholders of using smartwatches in response to the pandemic. In addition, social media is plagued with misinformation, which is beyond the scope of this research. It will be helpful to incorporate information credibility assessment as a preprocessing step to improve the quality of social media content for examining user experience with the smartwatch technology in future research. Despite these limitations, our study is the first study that uses a content analysis approach to understand the benefits and concerns of smartwatches in the context of this pandemic, which will help both practitioners and researchers better understand the rapidly growing device.

\section{Acknowledgment}

This research was partially supported by the National Science Foundation (Award \#s: CNS 1917537 and SES 1912898). Any opinions, findings, and conclusions, or recommendations expressed in this paper are those of the authors and do not necessarily reflect the views of the above funding agency.

\section{References}

[1] P.R. Center. "About one-in-five Americans use a smartwatch or fitness tracker,"

https://www.pewresearch.org/fact$\operatorname{tank} / 2020 / 01 / 09 /$ about-one-in-five-americans-use-asmart-watch-or-fitness-tracker/ (accessed May 14, 2021).

[2] Gartner. "Gartner forecasts global spending on wearable devices to total $\$ 81.5$ billion in 2021." https://www.gartner.com/en/newsroom/pressreleases/2021-01-11-gartner-forecasts-global-spendingon-wearable-devices-to-total-81-5-billion-in-2021 (accessed May 10, 2021) 
[3] J. Dunn, R. Runge, \& M. Snyder, "Wearables and the medical revolution," Personalized Medicine, vol. 15, no. 5, 2018, pp. 429-448.

[4] G. Quer, J.M. Radin, M. Gadaleta, K. Baca-Motes, L. Ariniello, E. Ramos, V. Kheterpal, E.J. Topol, \& S.R. Steinhubl., "Wearable sensor data and self-reported symptoms for COVID-19 detection," Nature Medicine, vol. 27 , no. 1 , 2021, pp. 73-77.

[5] T. Mishra, M. Wang, A.A. Metwally, G.K. Bogu, A.W Brooks, A. Bahmani, A. Alavi, A. Celli, E. Higgs, O. Dagan-Rosenfeld, \& B. Fay, "Pre-symptomatic detection of COVID-19 from smartwatch data," Nature Biomedical Engineering, vol. 4, no. 12, 2020, pp. 1208-1220.

[6] T. Zhu, P. Watkinson, \& D. A. Clifton, "Smartwatch data help detect COVID-19," Nature Biomedical Engineering, vol. 4 , no. 12,2020 , pp. 1125-1127.

[7] L.C. Ming, N. Untong, N.A. Aliudin, N. Osili, N. Kifli, C.S. Tan, K. W. Goh, P.W. Ng, Y.M. Al-Worafi, K.S. Lee, \& H.P. Goh, "Mobile health apps on COVID-19 launched in the early days of the pandemic: content analysis and review," JMIR mHealth and uHealth, vol. 8, no. 9, 2020, p. e19796.

[8] R. Collado-Borrell, V. Escudero-Vilaplana, C. Villanueva-Bueno, A. Herranz-Alonso, \& M. SanjurjoSaez, "Features and functionalities of smartphone apps related to COVID-19: systematic search in app stores and content analysis," Journal of Medical Internet Research, vol. 22 , no. 8, 2020, p. e20334.

[9] A. Bassi, S. Arfin, O. John, \& V. Jha, "An overview of mobile applications (apps) to support the coronavirus disease 2019 response in India," Indian Journal of Medical Research, vol. 151, no. 5, 2020, p. 468.

[10] M. Almalki \& A. Giannicchi, "Health apps for combating COVID-19: Descriptive review and taxonomy," JMIR mHealth and uHealth, vol. 9, no. 3, 2021, p. e24322.

[11] T. Alanzi, "A review of mobile applications available in the app and google play stores used during the COVID19 outbreak," Journal of Multidisciplinary Healthcare, vol. 14, 2021, p. 45.

[12] The DHS Science and Technology Directorate. "Nonmedical interventions: A desk reference to help planners recover from COVID-19 and prepare for future outbreaks and pandemics"

https://www.dhs.gov/sites/default/files/publications/202 0_0604_covid_19_interventions_rev14508.pdf (accessed May 30, 2021).

[13] C.H. Chau, J.D. Strope, \& W.D. Figg, "COVID-19 clinical siagnostics and testing technology," Pharmacotherapy: The Journal of Human Pharmacology and Drug Therapy, vol. 40, no. 8, 2020, pp. 857-868.

[14] S. Manigandan, P.K.T. Ramesh, N.T.L. Chi, \& K. Brindhadevi, "Early detection of SARS-CoV-2 without human intervention to combat COVID-19 using drone technology," Aircraft Engineering and Aerospace Technology, vol. 93, no. 1, 2020, pp. 85-88.

[15] M.N.K. Boulos \& E.M. Geraghty, "Geographical tracking and mapping of coronavirus disease COVID$19 /$ severe acute respiratory syndrome coronavirus 2 (SARS-CoV-2) epidemic and associated events around the world: how 21 st century GIS technologies are supporting the global fight against outbreaks and epidemics," International Journal of Health Geographics, 19, no. 8, 2020, doi: https://doi.org/10.1186/s12942-02000202-8.

[16] R.A. Kleinman \& C. Merkel, "Digital contact tracing for COVID-19," CMAJ, vol. 192, no. 24, 2020, pp. E653E656.

[17] L. Li, Z. Yang, Z. Dang, C. Meng, J. Huang, H. Meng, D. Wang, G. Chen, J. Zhang, H. Peng, \& Y. Shao, "Propagation analysis and prediction of the COVID-19," Infectious Disease Modelling, vol. 5, 2020, pp. 282-292.

[18] M.H. Gedde, B.S. Husebo, A. Erdal, N.G. Puaschitz, M. Vislapuu, R.C. Angeles, \& L.I. Berge, "Access to and interest in assistive technology for home-dwelling people with dementia during the COVID-19 pandemic (PAN. DEM)," International Review of Psychiatry, 2020, pp. 18.

[19] R. Jiwani, B. Dennis, C. Bess, S. Monk, K. Meyer, J. Wang, \& S. Espinoza, "Assessing acceptability and patient experience of a behavioral lifestyle intervention using fitbit technology in older adults to manage type 2 diabetes amid COVID-19 pandemic: A focus group study," Geriatric Nursing, vol. 42, no. 1, 2021, pp. 57-64.

[20] C. B. Taylor, E. E. Fitzsimmons-Craft, \& A. K. Graham, "Digital technology can revolutionize mental health services delivery: The COVID-19 crisis as a catalyst for change,"International Journal of Eating Disorders, vol. 53, no. 7, 2020, pp. 1155-1157.

[21] A. Busse W. Kashino, S. Suhartono, N. Narotama, D. Pelupessy, A.A. Fikri, \& C.A. Essau, "Acceptability and feasibility of using digital technology to train community practitioners to deliver a family-based intervention for adolescents with drug use disorders during the COVID19 pandemic," Addictive Behaviors Reports, 2021, p. 100357.

[22] C. Berardi, M. Antonini, M.G. Genie, G. Cotugno, A. Lanteri, A. Melia, \& F. Paolucci, "The COVID-19 pandemic in Italy: policy and technology impact on health and non-health outcomes," Health Policy and Technology, vol. 9, no. 4, 2020, pp. 454-487

[23] L. Ferretti C. Wymant, M. Kendall, L. Zhao, A. Nurtay, L. Abeler-Dörner, M. Parker, D. Bonsall, \& C. Fraser, "Quantifying SARS-CoV-2 transmission suggests epidemic control with digital contact tracing," Science, vol. 368 , no. $6491,2020$.

[24] A.F. Leite-Moreira \& R. Adao, "Mobile cardiology during the COVID-19 outbreak," Cardiovascular Research, vol. 116, no. 12, 2020, pp. e149-e151.

[25] J. Abbasi, "Using smartwatch data to detect COVID-19 cases early," JAMA, vol. 324, no. 22, 2020, pp. 22472247.

[26] E. Seto, P. Challa, \& P. Ware, "Adoption of COVID-19 Contact Tracing Apps: A Balance Between Privacy and Effectiveness," Journal of Medical Internet Research, vol. 23, no. 3, 2021, p. e25726.

[27] H. J. L. Singh, D. Couch, \& K. Yap, "Mobile health apps that help with COVID-19 management: scoping review," Jmir Nursing, vol. 3, no. 1, 2020, p. e20596.

[28] J.-P. Weiß, M. Esdar, \& U. Hübner, "Analyzing the Essential Attributes of Nationally Issued COVID-19 Contact Tracing Apps: Open-Source Intelligence 
Approach and Content Analysis," JMIR mHealth and uHealth, vol. 9, no. 3, 2021, p. e27232.

[29] K. Kaspar, "Motivations for social distancing and app use as complementary measures to combat the COVID-19 pandemic: quantitative survey study," Journal of Medical Internet Research, vol. 22, no. 8, 2020, p. e21613.

[30] A. Oldeweme, J. Märtins, D. Westmattelmann, \& G. Schewe, "The role of transparency, trust, and social influence on uncertainty reduction in times of pandemics: empirical study on the adoption of COVID-19 tracing apps," Journal of Medical Internet Research, vol. 23, no. 2, 2021, p. e25893.

[31] M. Elkhodr, O. Mubin, Z. Iftikhar, M. Masood, B. Alsinglawi, S. Shahid, \& F. Alnajjar, "Technology, privacy, and user opinions of covid-19 mobile apps for contact tracing: systematic search and content analysis," Journal of Medical Internet Research, vol. 23, no. 2, 2021, p. e23467.

[32] B. Sudharsan, D. Sundaram, J.G. Breslin, \& M.I. Ali, "Avoid touching your face: A hand-to-face $3 \mathrm{~d}$ motion dataset (covid-away) and trained models for smartwatches," in 10th International Conference on the Internet of Things Companion, 2020, pp. 1-9.

[33] S. Samyoun, S.S. Shubha, M.A.S. Mondol, \& J.A. Stankovic, "iWash: A smartwatch handwashing quality assessment and reminder system with real-time feedback in the context of infectious disease," Smart Health, vol. 19, 2021, p. 100171

[34] M. Strik, T. Caillol, F.D. Ramirez, S. Abu-Alrub, H. Marchand, N. Welte, P. Ritter, M. Haïssaguerre, S. Ploux, \& P. Bordachar, "Validating QT-interval measurement using the apple watch ECG to enable remote monitoring during the COVID-19 pandemic," Circulation, vol. 142, no. 4, 2020, pp. 416-418.

[35] J.S. Chinitz, R. Goyal, D.C. Morales, M. Harding, S. Selim, \& L.M. Epstein, "Use of a smartwatch for assessment of the QT interval in outpatients with coronavirus disease 2019," The Journal of innovations in cardiac rhythm management, vol. 11, no. 9, 2020, p. 4219

[36] X. Liu, J. Fan, Y. Guo, H. Dai, J. Xu, L. Wang, P. Hu, X. Lin, C. Li, D. Zhou, \& H. Li, "Smartwatch facilitated remote health care for patients undergoing transcatheter aortic valve replacement amid covid-19 pandemic," medRxiv, 2021.

[37] H. Niela-Vilén, J. Auxier, E. Ekholm, F. Sarhaddi, M. Asgari Mehrabadi, A. Mahmoudzadeh, I. Azimi, P. Liljeberg, A.M. Rahmani, A. Axelin, "Pregnant women's daily patterns of well-being before and during the COVID-19 pandemic in Finland: Longitudinal monitoring through smartwatch technology," PloS one, vol. 16, no.2, 2021, p. e0246494.

[38] K. Ueafuea, C. Boonnag, T. Sudhawiyangkul, P. Leelaarporn, A. Gulistan, W. Chen, S.C. Mukhopadhyay, T. Wilaiprasitporn, \& S. Piyayotai, "Potential applications of mobile and wearable devices for psychological support during the COVID-19 pandemic: A review," IEEE Sensors Journal. vol. 21, no. 6, 2020, pp. $7162-7178$.

[39] T. Ammari, S. Schoenebeck, \& D. M. Romero, "Pseudonymous parents: Comparing parenting roles and identities on the Mommit and Daddit subreddits," in Proceedings of the 2018 CHI Conference on Human Factors in Computing Systems, 2018, pp. 1-13.

[40] I. Mansoor, "Twitter Revenue and Usage Statistics (2021)," https://www.businessofapps.com/data/twitterstatistics/ (accessed May 15, 2021).

[41] D. Curry. "Reddit Revenue and Usage Statistics (2021)." https://www.businessofapps.com/data/reddit-statistics/ (accessed May 17, 2021).

[42] S. Aggarwal, N. Garcia-Telles, G. Aggarwal, C. Lavie, G. Lippi, \& B.M. Henry, "Clinical features, laboratory characteristics, and outcomes of patients hospitalized with coronavirus disease 2019 (COVID-19): early report from the United States," Diagnosis, vol. 7, no. 2, 2020, pp. 91-96.

[43] A.M. Bunting, D. Frank, J. Arshonsky, M.A. Bragg, S.R. Friedman, \& N. Krawczyk, "Socially-supportive norms and mutual aid of people who use opioids: An analysis of Reddit during the initial COVID-19 pandemic," Drug and alcohol dependence, vol. 222, 2021, p. 108672.

[44] K. Kousha \& M. Thelwall, "COVID-19 publications: Database coverage, citations, readers, tweets, news, Facebook walls, Reddit posts," Quantitative Science Studies, vol. 1, no. 3, 2020, pp. 1068-1091.

[45] B. Downe-Wamboldt, "Content analysis: method, applications, and issues," Health care for women international, vol. 13, no. 3, 1992, pp. 313-321.

[46] Stemler, Steven E. "Content analysis," Emerging trends in the social and behavioral sciences: An Interdisciplinary, Searchable, and Linkable Resource, 2015, pp. 1-14.

[47] M. Williams \& T. Moser. "The art of coding and thematic exploration in qualitative research." International Management Review 15, no. 1, 2019, pp. 45-55.

[48] K.L. Gwet, "Computing inter-rater reliability and its variance in the presence of high agreement," British Journal of Mathematical and Statistical Psychology, vol. 61, no. 1, 2008, pp. 29-48.

[49] H. Jelodar, Y. Wang, C. Yuan, X. Feng, X. Jiang, Y. Li, \& L. Zhao. "Latent Dirichlet allocation (LDA) and topic modeling: models, applications, a survey," Multimedia Tools and Applications, vol. 78, no. 11, 2019, pp. 1516915211.

[50] D. Maier, A. Waldherr, P. Miltner, G. Wiedemann, A. Niekler, A. Keinert, B. Pfetsch, G. Heyer, U. Reber, T. Häussler, H. Schmid-Petri, \& S. Adam. "Applying LDA topic modeling in communication research: Toward a valid and reliable methodology," Communication Methods and Measures, vol. 12, no. 2-3, 2018, pp. 93118.

[51] X. Wang, A. McCallum, \& X. Wei, "Topical n-grams: Phrase and topic discovery, with an application to information retrieval," in Seventh IEEE international conference on data mining, 2007: IEEE, pp. 697-702.

[52] A.J. Perez. "Use a fitness app to track your workouts? Your data may not be as protected as you think." https://www.usatoday.com/story/sports/2019/08/16/what -info-do-fitness-apps-keep-share/1940916001/ (accessed May 1, 2021). 